\title{
Compartment Syndrome of the Upper Extremity Induced by Extravasation of Contrast Media after Computed Tomography: A Case Report
}

\author{
Jae-Won Jung, Young-Jae Lim, Beom-Soo Kim, Chul-Hyun Cho \\ Department of Orthopedic Surgery, Dongsan Medical Center, Keimyung University School of Medicine, Daegu, Korea
}

\begin{abstract}
The incidence of contrast media extravasation has been increasing gradually. Most contrast media extravasations only can cause pain, swelling and erythema. However, in more severe cases, skin necrosis, ulceration, or compartment syndrome may occur, often necessitating a surgery. Early diagnosis and treatment should be established on the spot in order to avoid such a severe damage. We present a case of 42-year-old woman with a compartment syndrome in the right upper extremity caused by extravasation of computed tomography contrast media.
\end{abstract}

(Clin Shoulder Elbow 2017;20(4):240-243)

Key Words: Upper extremity; Contrast media; Compartment syndrome

Advancements in radiological technology has led to more accurate and early diagnosis for various diseases. However, because many of the radiological examinations require patients to take contrast media, it has led to the increase in the prevalence of complications such as extravasation into the surrounding tissue. In its mild form, extravasation of contrast media can lead to mild pain, swelling, or redness in patients. But in its severe form, it can lead to skin necrosis, ulcers, or compartment syndrome. Therefore, early diagnosis and treatment is necessary for these complications following contrast media extravasation." Previous studies have reported that extravasation induced complications after injection of contrast media occur in $0.25 \%$ to $0.9 \%$ of patients. $^{2-4)}$ Although a few studies have described the occurrence and surgical treatment of compartment syndrome after contrast media extravasation, those reporting compartment syndrome of the hand let alone the entire forearm are very rare. Therefore, the authors report a case of compartment syndrome of the right upper extremity caused by extravasation of contrast media in a 42-year-old female patient. Along with a review of the current literature regarding this syndrome, we report the favorable outcomes of the surgical treatment of contrast media extravasation in this patient.

\section{Case Report}

This study has been exempted from informed consent of Institutional Review Board (IRB File No. DSMC 2017-11-009).

A 42-year-old female patient with a body mass index (BMI) of $36.1 \mathrm{~kg} / \mathrm{m}^{2}$ who had treated for uterine endometrial cancer visited hospital to evaluate the possibility of metastasis. Computed tomography of the chest and abdomen was scheduled for the patient. In AM 9, after receiving an intravenous injection of contrast media into the dorsum of the right hand, the patient presented with pain and swelling in the hand and wrist. The contrast media used during the computed tomography was 300 $\mathrm{mg} \mathrm{I/ml} \mathrm{of} \mathrm{Ultravist} \mathrm{(Iopromide;} \mathrm{Bayer} \mathrm{Schering,} \mathrm{Berlin,} \mathrm{Ger-}$ many). An automated injector was used to inject a total of 60 $\mathrm{ml}$ of the material at a rate of $2.5 \mathrm{ml} / \mathrm{sec}$ with 20-gage needle.

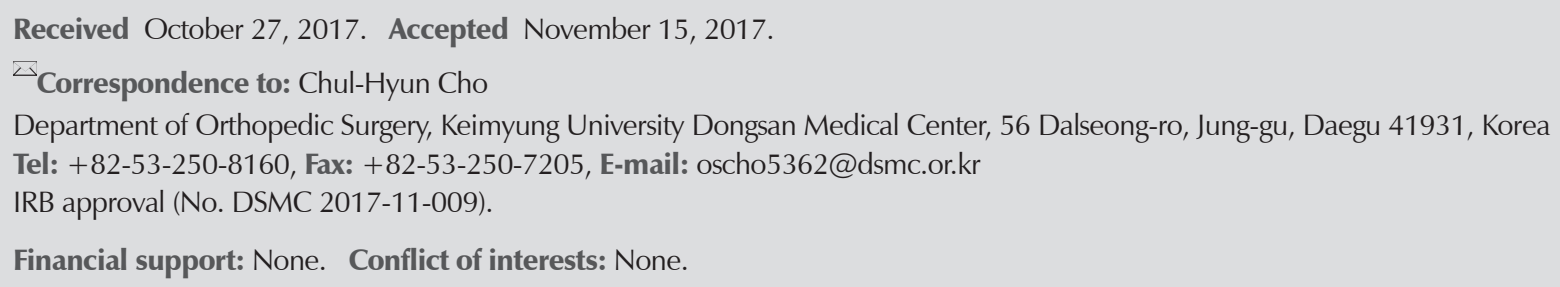




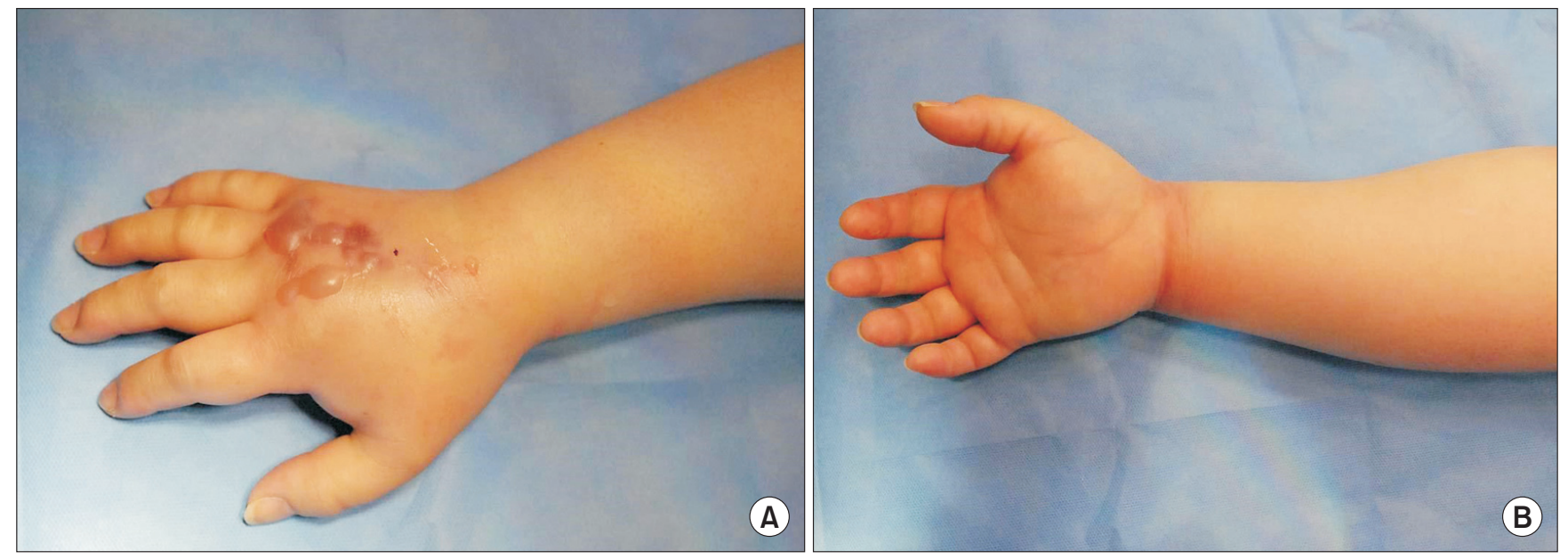

Fig. 1. Clinical findings of extensive swelling and bullae formation on the right hand (A) and forearm (B).
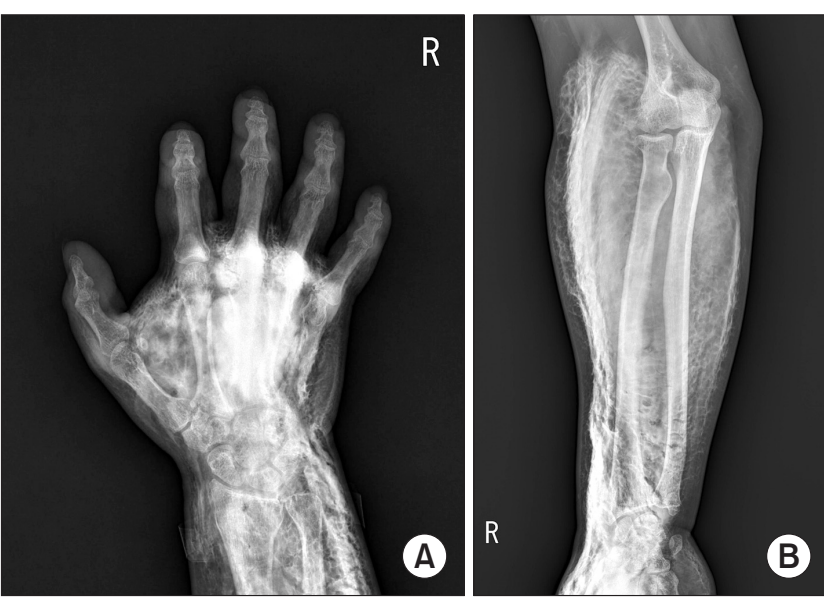

Fig. 2. Simple radiographs showing extensive infiltration of the contrast media stretching from the hand (A) to the elbow (B) after computed tomography.

The patient complained of the pain to the radiologist soon after the injection, but the patient was discharged after being advised that the pain would be temporary. In AM 10, the initial pain in the right hand and wrist became severe after time, leading to swelling and redness in the forearm. In AM 11, she visited the dermatologic department and consulted to the orthopedic department because of these symptoms. In PM 2:30, the patient was suspected of having compartment syndrome induced by extravasation of the contrast media and was sent to the emergency room immediately. The patient presented with unstable vital signs: blood pressure of $174 / 127 \mathrm{mmHg}$, a heart rate of 120 beats/min, and a body temperature of $37.2^{\circ} \mathrm{C}$. Physical examination showed multiple bullae formation on the dorsum of the right hand and wrist; swelling and redness from the hand to the distal forearm; and severe pain during finger extension (Fig. 1). Simple radiograph showed the contrast media extravasation that expanded from the right hand to the distal upperarm (Fig. 2).

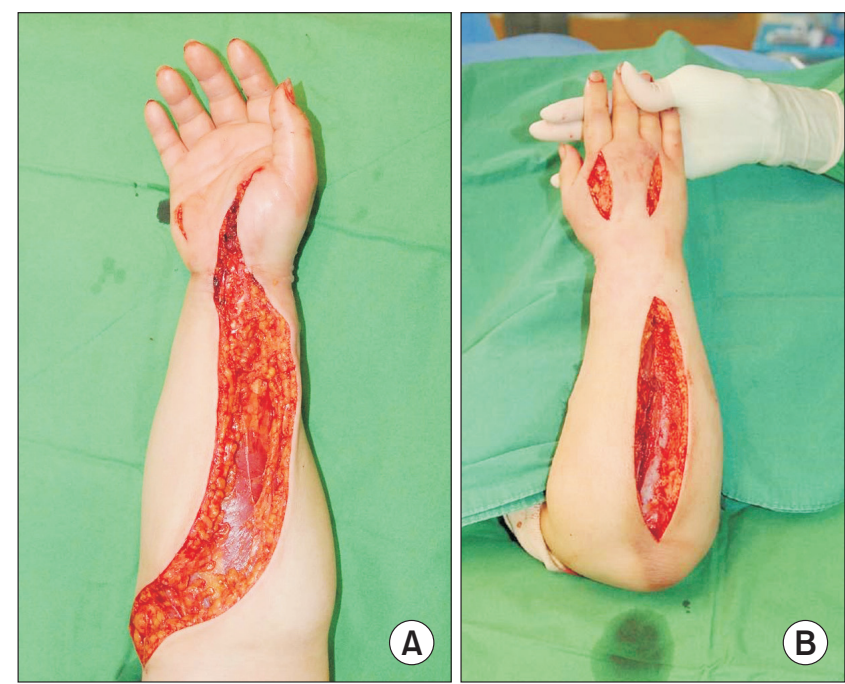

Fig. 3. Intraoperative images of the fasciotomy of the right hand and forearm: the volar side (A) and the dorsal side (B).

Blood tests did not show abnormal findings. On the basis of the clinical and radiological findings, she was diagnosed as a compartment syndrome without measuring the pressure of the forearm. In PM 4, the patient was moved to operation room and took an emergent decompressive fasciotomy under general anesthesia (Fig. 3). The decompressive fasciotomy was performed on the anteroposterior hand and forearm with the wound was left open. The patient's pain and swelling was relieved immediately after surgery and resolved by the fourth postoperative day, as well as the active and passive range of motions of the fingers. After that we closed the surgical wound. With complete resolution of the symptoms, the patient was discharged after 18 days of the treatment. At the time of discharge, the patient was able to perform passive and active extensions of the right wrist and fingers. At the 1-year follow-up, we found that the function of 
the wrist and fingers was restored, but patient was dissatisfied with scar wound cosmetically.

\section{Discussion}

As the increase of radiologic examination using contrast media, complications associated with contrast media is increasing such as extravasation. It has been reported that extravasation-related complications occur in $0.25 \%$ to $0.9 \%$ of patients injected with contrast media. ${ }^{2-4)}$ Wang et al. ${ }^{1)}$ had reported that extravasation of contrast media cause mild pain, swelling, and redness and that they can be conservatively treated with favorable outcomes. On the other hand, some case reports for more severe complications, such as compartment syndrome have shown that surgical treatment within 6 hours of exposure is essential to prevent extensive injury. ${ }^{5,6)}$

The extent of tissue injury induced by contrast media extravasation has been shown to depend on the type, volume, and osmolarity of the contrast media and on the injection route and speed. ${ }^{5)}$ Miles et al. ${ }^{6}$ reported that more than $50 \mathrm{ml}$ of extravasation after computed tomography leads to an increased chance of complications such as compartment syndrome. Sbitany et al. ${ }^{7}$ defined a large volume of extravasation as $>150 \mathrm{ml}$ and reported that any surgical treatment is needed in case of exceeding that volume. In the past, most tissue damage was related to extravasation of hyperosmotic, ionic contrast media, but after use of hypoosmotic, non-ionic contrast media, the incidence of tissue damage has been decreased recently. ${ }^{78)}$ However, increasing number of intravenous injection to the distal portion of extremity or the automated injection of the contrast media has higer risk of extravasation, it should be used with caution. Injection sites such as lower extremity or small vein, and patient factors such as diabetes, cancer, obesity have been reported to risk factor of extravasation. ${ }^{1,8)}$

There is no standardized treatment for injuries resulting from the extravasation of contrast media. Standard early interventions include keeping the extremity damaged by extravasation more elevated than the heart level, applying an ice-pack to reduce the swelling and redness; and immobilization, with considering possibility of compartment syndrome. In the Korean literature, a number of studies have shown that conservative treatment alone can lead to favorable outcomes for compartment syndrome. For instance, Shin et al..$^{8)}$ reported that conservative treatment including elevation of the area of extravasation, simple compression, ice-pack application, and hand grip exercise was associated with favorable outcomes for compartment syndrome. Similarly, Huh et al. ${ }^{9)}$ reported that symptoms of compartment syndrome was relieved after the administration of preventative antibiotics under upper extremity elevation and long-arm cast immobilization. Nevertheless, surgical treatment including fasciotomy is effective and has shown satisfactory outcome for compartment syndrome. ${ }^{6}$ But, the most important thing is prevention against contrast media extravasation and close observation of pain and swelling for the early detection. ${ }^{10)}$

In this case report, we reported the use of non-ionic, watersoluble contrast media in a obese patient with a BMI of 36.1 $\mathrm{kg} / \mathrm{m}^{2}$. The patient who had had repetitive chemotherapy to treat her uterine endometrial cancer had poor vasculature. We presumed that a vascular tear may have been caused when an automated injector was used to intravenously inject the contrast media and extravasation had occured. Despite the patient's complaint of pain and swelling soon after the contrast media injection, the medical staff, such as doctors, nurses, and radiologists failed to respond with the appropriate management processes. Based on clinical symptoms such as severe swelling and pain in the hand and forearm, which spread to the elbow within a short period of time. Although compartment syndrome was diagnosed and surgical treatment was performed after 6 hours of exposure to the contrast media, we achieved favorable outcome in this patient. The limitations of this study are that we did not measure the interstitial pressure in spite of a clinical diagnosis of compartment syndrome.

An accurate diagnosis through early clinical and radiological assessments is essential, and future studies investigating the standard treatment for contrast media extravasation-induced compartment syndrome are required.

\section{References}

1. Wang $\mathrm{CL}$, Cohan $\mathrm{RH}$, Ellis JH, Adusumilli $\mathrm{S}$, Dunnick NR. Frequency, management, and outcome of extravasation of nonionic iodinated contrast medium in 69,657 intravenous injections. Radiology. 2007;243(1):80-7.

2. Cohan RH, Bullard MA, Ellis JH, et al. Local reactions after injection of iodinated contrast material: detection, management, and outcome. Acad Radiol. 1997;4(11):711-8.

3. Federle MP, Chang PJ, Confer S, Ozgun B. Frequency and effects of extravasation of ionic and nonionic CT contrast media during rapid bolus injection. Radiology. 1998;206(3):637-40.

4. Jacobs JE, Birnbaum BA, Langlotz CP. Contrast media reactions and extravasation: relationship to intravenous injection rates. Radiology. 1998;209(2):411-6.

5. Belzunegui T, Louis CJ, Torrededia L, Oteiza J. Extravasation of radiographic contrast material and compartment syndrome in the hand: a case report. Scand J Trauma Resusc Emerg Med. 2011;19:9.

6. Miles SG, Rasmussen JF, Litwiller T, Osik A. Safe use of an intravenous power injector for CT: experience and protocol. Radiology. 1990;176(1):69-70.

7. Sbitany H, Koltz PF, Mays C, Girotto JA, Langstein HN. CT contrast extravasation in the upper extremity: strategies for management. Int J Surg. 2010;8(5):384-6. 
8. Shin DJ, Byun YS, Choi S. Extravasation injury of forearm by computed tomography contrast medium. J Korean Orthop Assoc. 2013;48(1):27-32.

9. Huh SW, Ryu JH, Song SW, Rhee SK. Compartment syndrome of forearm caused by extravasation of ct contrast media: a case report. J Korean Soc Surg Hand. 2012;17(1):43-6.

10. Kim HI, Shin DA. Automated pressure-controlled discography with constant injection speed and real-time pressure measurement. J Korean Neurosurg Soc. 2009;46(1):16-22. 